\title{
Results on search for new physics with kaons
}

\author{
Riccardo Fantechi \\ on behalf of the NA62 Collaboration \\ INFN - Sezione di Pisa \\ Largo B. Pontecorvo 3, I56127 Pisa
}

\begin{abstract}
The ratio $R_{K}=\Gamma\left(K^{ \pm} \rightarrow e^{ \pm} \nu\right) / \Gamma\left(K^{ \pm} \rightarrow \mu^{ \pm} \nu\right)$ is predicted with excellent precision by the Standard Model. In a recent paper, it has been pointed that SUSY extensions to the SM can introduce a shift of a few percent in $\mathrm{R}_{K}$, due to $\mu$-e violation. The average of all the measurements up to now, including preliminary ones by NA48 and KLOE, has a relative error on $\mathrm{R}_{K}$ of $1.5 \%$ : a better measurement could be very interesting to test the SM. The NA62 Collaboration took data in 2007 to measure $\mathrm{R}_{K}$ in order to achieve a relative error of better than $0.5 \%$. About $160 \mathrm{~K} K^{ \pm} \rightarrow e^{ \pm} \nu$ events has been collected, together with data from special runs to study systematic effects. An analysis, based on $40 \%$ of the total statistics, is almost completed: the relative error is $0.6 \%$ and the preliminary result for $R_{K}$ will be presented in 2009 summer conferences.
\end{abstract}

\section{Lepton Flavor Violation}

The ratio $\mathrm{R}_{K}$ between the two leptonic decays of the charged kaon is known very precisely, because of the cancellation of the hadronic contributions in the form factor of the $\mathrm{K}$. The actual estimation [1] is:

$$
R_{K}=\frac{\Gamma\left(K^{ \pm} \rightarrow e^{ \pm} \nu\right)}{\Gamma\left(K^{ \pm} \rightarrow \mu^{ \pm} \nu\right)}=\left(\frac{m_{e}}{m_{\mu}}\right)^{2}\left(\frac{m_{K}^{2}-m_{e}^{2}}{m_{K}^{2}-m_{\mu}^{2}}\right)^{2}\left(1+\delta R_{Q E D}\right)=(2.477 \pm 0.001) \cdot 10^{-5}
$$

The small rate of the electronic decay respect to the muonic one is due to helicity suppression. A non negligible contribution to the theoretical estimation is due to the radiative $K_{l 2 \gamma}$ decay. The inner bremsstrahlung and virtual photon processes are taken into account and included in the $\delta R_{Q E D}=-3.4 \%$ correction, while the structure dependent part is considered as background.

The Standard Model strongly suppresses Lepton Flavour Violation (LFV). However, it has been shown in [2] that LFV contribution can arise in Supersymmetric extensions of the SM: here deviations of $R_{K}$ up to few $\%$ with respect to the SM predictions can be present without contradicting any present experimental constraints. Evidence for new physics beyond the SM could then be shown with a precise measurement of $R_{K}$.

\section{Experimental status}

Up to 2006 the world average for $R_{K}$ was based on results from experiments performed in $1970 \mathrm{~s}$. The value was $R_{K}=(2.45 \pm 0.11) \cdot 10^{-5}$. The relative error $\delta R_{K} / R_{K}=4.5 \%$ was too high for any possible search of new physics beyond the SM. Recently, two new measurements from NA48/2 [3,4] and from KLOE [5] have been available. A combined result was presented by the FLAVIANET working group: $R_{K}=(2.457 \pm 0.032) \cdot 10^{-5}$, with a relative error of $1.5 \%$. The value is in agreement with the SM, but a better measurement is needed for a stringent test. In winter 2009, KLOE presented its final result [6], based on $14 \mathrm{~K} K_{e 2}$ candidates: $R_{K}=(2.493 \pm 0.025 \pm 0.019) \cdot 10^{-5}$ with a relative error of $1.3 \%$.

DIS 2009 


\section{The detector}

In 2007 the NA62 Collaboration has used the NA48 detector to collect data for the measurement of $R_{K}$. During about 120 days of data taking, some $90 \mathrm{~TB}$ of data have been put on tape and about $160 \mathrm{~K} K_{e 2}$ decays are available for the analysis, aiming to a measurement of $R_{K}$ with a total relative error less than $0.5 \%$. Special runs have been performed also in 2008 to control various possible systematic contributions at the same level of the statistical error.

The beam line design is derived by the NA48 one: $400 \mathrm{GeV} / \mathrm{c}$ protons on a Be target generate high intensity charged kaons of both polarity. A system of magnetic achromats select a kaon beam with a momentum of $75 \pm 2 \mathrm{GeV} / \mathrm{c}$, with a kaon/pion ratio of 1:9. Despite the availability of both charges, the majority of the data has been collected with the positive charge, as the negative one contains a greater background contribution from the beam halo. The kaon momentum is not measured directly event by event, but the average value is obtained by $K^{+} \rightarrow \pi^{+} \pi^{+} \pi^{-}$decays and its spread is $\Delta P_{K}^{R M S} / P_{K}=2 \%$.

The significant components of the detector (fully described in [7]) are:

- The magnetic spectrometer, composed of 4 drift chambers and a magnet. Each chamber is composed of eight planes of sense wires arranged in four pairs of staggered "views", oriented at 0,90 and \pm 45 degrees. The resolution of the spectrometer is $\delta_{p} / p=0.47 \% \oplus 0.020 \% p$, with $p$ expressed in $\mathrm{GeV} / \mathrm{c}$.

- A plastic scintillator hodoscope, used to produce fast trigger signals. It is made up of 64 horizontal and 64 vertical scintillator strips arranged in four quadrants.

- A liquid krypton electromagnetic calorimeter, an almost-homogeneous ionization chamber with an active volume of $7 \mathrm{~m}^{3}$ of liquid krypton, segmented transversally in 13248 projective, $2 \times 2 \mathrm{~cm}^{2}$ cells with a system of ribbon electrodes and no longitudinal segmentation. The energy resolution is $\sigma_{E} / E=3.2 \% / \sqrt{E} \oplus 9 \% E \oplus 0.42 \%$, with $E$ in $\mathrm{GeV}$.

\section{The trigger}

It has been chosen to run with a low bias trigger. The initial setup for the $K_{e 2}$ trigger was the coincidence of hits between two hodoscope planes (Q1) and the presence of at least 10 $\mathrm{GeV}$ of energy in the calorimeter, while the $K_{\mu 2}$ trigger was based on the Q1 condition alone and a downscaling factor of 50 . Then, to better define the fiducial volume, a condition on the presence of at least one track in the drift chamber has been added to both the trigger conditions. Other downscaled trigger conditions were used to collect control samples to monitor the trigger inefficiencies. The Q1 inefficiency cancels out in the ratio, while the one for the energy condition is controlled with the downscaled Q1 sample and it is less than $0.1 \%$ with a cut of $15 \mathrm{GeV} / \mathrm{c}$ on the track momentum. During the last part of the run, the maximum bandwidth of about $55 \mathrm{~K}$ triggers per SPS spill was obtained and the number of $K_{e} 2$ candidates/spill was on average 0.54 .

\section{The measurement strategy}

The measured value of $R_{K}$ is defined as: $R_{K}=\frac{1}{D} \frac{N\left(K_{e 2}\right)-N_{b}\left(K_{e 2}\right)}{N\left(K_{\mu 2}\right)-N_{b}\left(K_{\mu 2}\right)} \frac{f_{\mu}}{f_{e}} \frac{A\left(K_{\mu 2}\right)}{A\left(K_{e 2}\right)} \frac{\epsilon\left(K_{\mu 2}\right)}{\epsilon\left(K_{e 2}\right)}$

DIS 2009 
where $N\left(K_{l 2}\right)$ are the numbers of the selected candidates and $N_{B}\left(K_{l 2}\right)$ those of the corresponding background events, $f_{l}$ are the identification efficiencies, $A\left(K_{l 2}\right)$ are the geometric acceptances computed with MC, $\epsilon\left(K_{l 2}\right)$ the trigger efficiencies and D is the downscaling factor for the $K_{\mu 2}$ trigger.

All these quantities can be evaluated from the data. Both decays are collected simultaneously, so the result is independent from the beam flux and a lot of systematics, as detector and trigger efficiencies, cancel out in the ratio. The ratio $R_{K}$ is evaluated in bins of the lepton momentum, mainly to control the muon contamination in the electron sample.

The selection of the two decays is based on kinematics and particle identification. The requirement that the reconstructed missing mass $M_{m i s s}^{2}=\left(P_{K}-p_{l}\right)^{2}$ is compatible with zero, allows the selection of the two leptonic decays. The ratio of the energy measured in the calorimeter and the momentum from the spectrometer $(\mathrm{E} / \mathrm{p})$ is used to identify electrons $(0.95<E / p<1.10)$ and muons $(E / p<0.2)$. An important issue for the analysis is the probability for a muon to fake an electron.

The results presented here are based on $40 \%$ of the total statistics.

\section{Systematic uncertainties}

\subsection{Contaminations in the $K_{e 2}$ sample}

The main source of this background is the $K_{\mu 2}$ decay, where a muon is misidentified as an electron: this could happen when a muon releases almost all its energy in the LKr calorimeter (catastrophic bremsstrahlung) and then it is identified as an electron. The probability of such an event is $\approx 3 \cdot 10^{-6}$, which leads, given $K_{\mu 2} / K_{e 2} \approx 40000$, to a misidentification $\mathrm{O}(10 \%)$. At momenta smaller than $35 \mathrm{GeV} / \mathrm{c}$, the $M_{m i s s}^{2}$ variable, evaluated with the electron mass hypothesis, could be used for electron-muon separation (Fig. 1). Above that value, one has to rely on $\mathrm{E} / \mathrm{p}$ and in this case catastrophic bremsstrahlung is an issue. The probability of this effect could be computed by Montecarlo, but to crosscheck this evaluation, NA62 has measured it directly with the data. During a part of data taking, a $4.5 \mathrm{~cm}$ thick lead wall was inserted between the two planes of the hodoscope, $1 \mathrm{~m}$ ahead of the calorimeter (with a $18 \%$ loss of acceptance).

All electrons in that region make a shower in the lead. An additional requirement on the pulse height on the second plane of the hodoscope selects pure muons in the calorimeter. A proper account is taken of the energy loss in the wall to reconstruct the energy of the muon. Additional pure muon samples come from special runs where the hadron beam was dumped and from $K_{\mu 2}$ decays during standard data taking. The distribution of $P(\mu \rightarrow e)$ is shown in fig. 2 and, after correcting for energy loss and muon bremsstrahlung in lead, the misidentification probability is $(7.4 \pm 0.2) \%$. The accuracy of this measurement will be improved with the analysis of a special run taken in 2008 with twice the amount of data with the lead wall.

Other backgrounds in the $K_{e 2}$ sample are:

- The effect of the muon halo of the beam, measured to be $(1.3 \pm 0.1) \%$ using data collected with the $K^{+}$beam dumped. Data from special runs in 2008 will improve the error.

- The structure dependent radiative $K_{e 2 \gamma}$ contamination is estimated to $(1.6 \pm 0.1) \%$, with the error dominated by the poor knowledge of the experimental value of the branching ratio. An NA62 ongoing analysis with 2007 data will improve it.

DIS 2009 


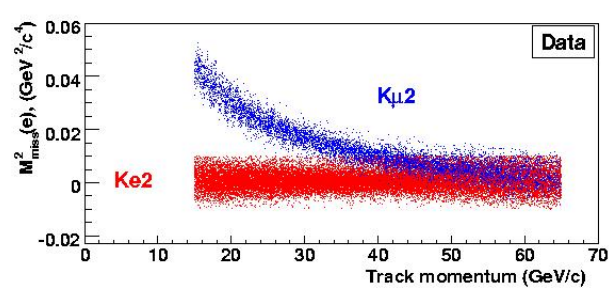

Figure 1: Missing mass squared assuming electron hypothesis for $K_{e 2}$ and for $K_{\mu 2}$ vs momentum

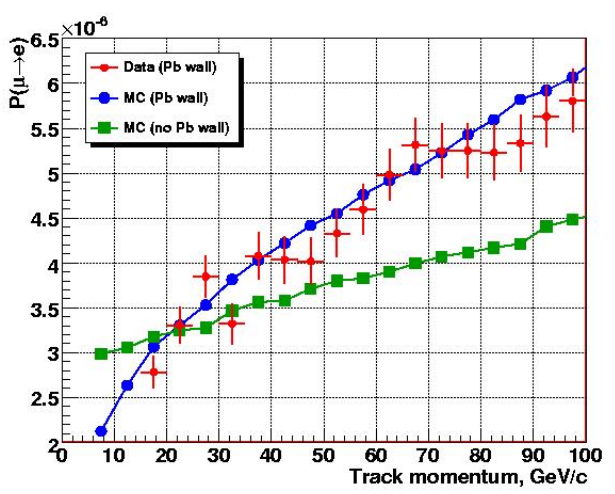

Figure 2: $P(\mu \rightarrow e)$ as a function of momentum

- $K_{\mu 2}$ events with a muon decay are included in the simulation and the correction is $(1.3 \pm 0.1) \%$.

\subsection{Backgrounds in the $K_{\mu 2}$ sample}

The background from the muon halo is around $0.2 \%$ and the contamination from $K^{+} \rightarrow$ $\pi^{+} \pi^{0}$ is below $0.5 \%$

\subsection{Efficiencies and acceptance}

\subsubsection{Electron identification efficiency}

The value and the energy dependence of the electron id efficiency $\left(f_{e}\right)$ can be measured with a clean sample of electrons from $K^{ \pm} \rightarrow \pi^{0} e^{ \pm} \nu$ decays collected with the main data sample. In this case, however, the electron momentum is limited to less than $50 \mathrm{GeV} / \mathrm{c}$. To cover all the interesting energy range, a special 15 hours run was performed using a broadband $K_{L}$ beam. The results is $f_{e}=99.15 \%$ with an uncertainty less than $0.1 \%$.

\subsubsection{Muon identification efficiency}

It is momentum dependent and varies between 0.996 and 0.999 and can be measured better than $0.1 \%$ with a pure muon sample

\subsubsection{Trigger efficiency correction}

The ratio of the two efficiency is measured directly from the data using control samples. Other known sources of systematic uncertainties are a possible bias of the downscaling factor due to Q1 afterpulses and the global calorimeter readout inefficiency.

\subsubsection{Geometric acceptance correction}

The ratio of the two geometric acceptances is momentum dependent and it varies between 1.2 and 1.3 

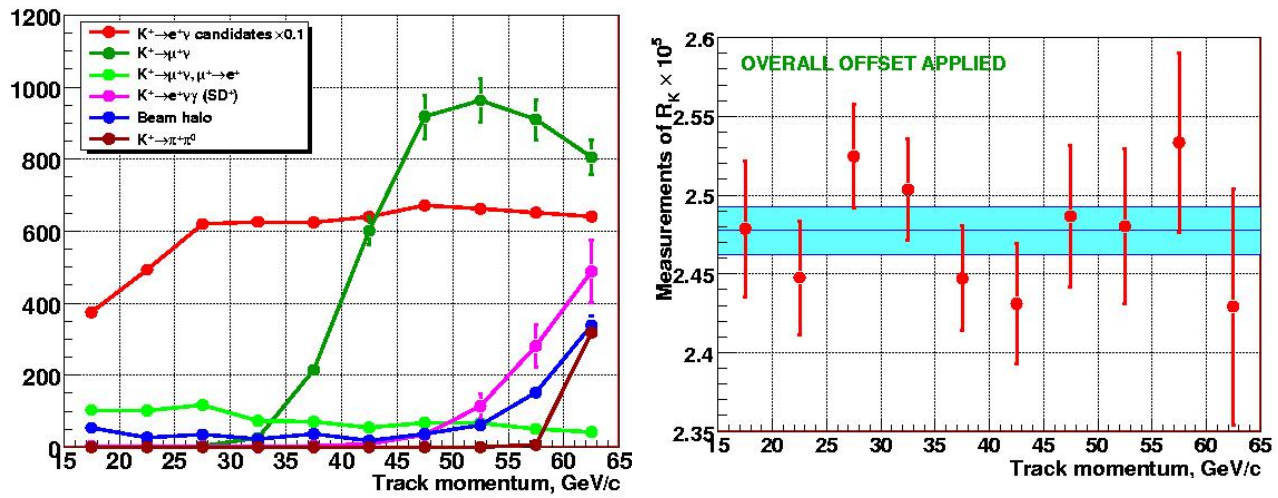

Figure 3: The different background contri- Figure 4: $R_{K}$ in momentum bins. An offbutions as a function of momentum set has been added to the true result

\section{$7 \quad$ Results}

Fig. 3 shows the momentum dependence of the various background contributions. Adding the various systematic erros to a statistical error of $0.4 \%$, we get a total error from this preliminary analysis of $0.6 \%$. Fig. 4 shows the uniformity of the $R_{K}$ value (with an offset added) as a function of the lepton momentum. There is still room to improve systematic errors. With the total statistics $\left(\approx 160 K K_{e 2}\right.$ candidates $)$, we plan to reach a statistical error of $0.3 \%$ and a total uncertainty of $0.4-0.5 \%$.

\section{Conclusions}

In 2007 NA62 has performed a 120 days run on the NA48/2 beam line to measure $R_{K}$ with a precision of better than $0.5 \%$. A preliminary analysis of $40 \%$ of the total statistics has shown that systematics are under control and can be improved in the full analysis. With this reduced sample, an accuracy of $0.6 \%$ has been achieved.

\section{References}

[1] V.Cirigliano, I. Rosell, Phys. Rev. Lett. 99231801 (2007)

[2] A. Masiero, P. Paradisi, R. Petronzio, Phys. Rev. D74 011701 (2006)

[3] L. Fiorini, PoS(HEP2005) 288 (2005)

[4] V. Kozhuharov, PoS(KAON) 049 (2007)

[5] F. Ambrosino et al., arXiv:0707.4623

[6] T. Spadaro, KLOE Collaboration, arXiv:0907.2613

[7] V. Fanti et al, Nucl. Instrum. Methods A574 (2007) 433

DIS 2009 